Journal of Sports Sciences 31, 434-441, 2013.

\title{
The influence of cricket fast bowlers' front leg technique on peak ground reaction forces
}

\author{
${ }^{1}$ P.J. Worthington, ${ }^{1}$ M.A. King and ${ }^{2}$ C.A. Ranson
}

${ }^{1}$ School of Sport, Exercise and Health Sciences, Loughborough University, Leicestershire, LE11 3TU, UK
${ }^{2}$ Cardiff School of Sport, Cardiff Metropolitan University, Cyncoed Campus, Cardiff, CF23 6XD

\begin{abstract}
High ground reaction forces during the front foot contact phase of the bowling action are believed to be a major contributor to the high prevalence of lumbar stress fractures in fast bowlers. This study aimed to investigate the influence of front leg technique on peak ground reaction forces during the delivery stride. Three-dimensional kinematic data and ground reaction forces during the front foot contact phase were captured for 20 elite male fast bowlers. Eight kinematic parameters were determined for each performance, describing run-up speed and front leg technique, in addition to peak force and time to peak force in the vertical and horizontal directions. There were substantial variations between bowlers in both peak forces (vertical $6.7 \pm 1.4$ body weights; horizontal (braking) $4.5 \pm 0.8$ body weights) and times to peak force (vertical $0.03 \pm 0.01 \mathrm{~s}$; horizontal $0.03 \pm$ $0.01 \mathrm{~s}$ ). These differences were found to be linked to the orientation of the front leg at the instant of front foot contact. In particular, a larger plant angle and a heel strike technique were associated with lower peak forces and longer times to peak force during the front foot contact phase, which may help reduce the likelihood of lower back injuries.
\end{abstract}

Keywords: cricket, fast bowling, peak forces, technique

\section{INTRODUCTION}

Cricket is generally considered to be a relatively low-injury sport, with only around five percent of elite players being unavailable to play due to injury at any given time (Orchard et al., 2006; Mansingh et al., 2006; Stretch, 2003; Newman, 2003). However, fast bowlers have injury prevalence of approximately $15 \%$ (Orchard et al 2006), rates similar to those reported for contact sports such as Australian Rules football (Orchard et al., 2002) and Rugby Union (Brooks et al., 2005). The most prevalent injury among fast bowlers is non-dominant side lumbar stress fracture (Orchard et al., 2006). High peak ground reaction forces during the front foot contact phase of the fast bowling action are believed to be a major contributor to these lower back injuries (Bartlett et al., 1996). These high peak forces coincide with the period of the action when lower trunk movements known to produce high contralateral facet joint contact forces occur (lower trunk extension in conjunction with contralateral sideflexion and ipsilateral rotation; Ranson et al., 2008; Burnett et al., 1998; Burnett et al., 2008).

A non-significant trend towards a faster rate of peak force development (in both the vertical and braking directions) during the front foot contact phase has been noted for a group of bowlers who had previously suffered a lower back stress fracture (Portus et al., 2004). Peak ground reaction forces during the front foot contact phase vary widely between bowlers, with values of $3.8-9.0$ bodyweights (BW) vertically and peak braking forces of $1.4-4.5 \mathrm{BW}$ being reported (Hurrion et al., 2000). The most recent studies (Hurrion et al., 2000; Portus et al., 2004) have observed higher peak braking forces (4.5 BW) than those reported previously. A quicker run-up speed, bowling technique, or the commitment and ease with which bowlers were able to bowl within the confines of the testing procedure have been suggested as explaining these higher peak forces (Hurrion et al., 2000). 
The motion of the front leg during the front foot contact phase has been implicated as a mechanistic factor in the development of lower back injury in fast bowlers (Foster et al., 1989; Mason et al., 1989, as cited by Portus et al., 2004). This led to Portus et al. (2004) dividing front leg technique into four categories based on the amount of knee flexion / extension during the front foot contact phase. An apparently arbitrary value of $10^{\circ}$ was chosen as the cut-off between categories, which were defined as: flexor, $10^{\circ}$ or more knee flexion followed by less than $10^{\circ}$ knee extension; flexor-extender, knee flexion and extension of $10^{\circ}$ or more; extender, less than $10^{\circ}$ knee flexion followed by $10^{\circ}$ or more knee extension and constant brace, less than $10^{\circ}$ knee flexion / extension. Techniques comprising front knee flexion during the front foot contact phase have been recommended in order to dissipate ground reaction forces and reduce the likelihood of injury (Foster et al., 1989). This has been supported by a study of eight English club fast and medium pace bowlers (Hall, 1999).

Bowlers with a flexed front knee at the instant of front foot contact tend to have lower peak ground reaction forces than those with a more extended front knee (Elliott, 2000). Similarly, Portus et al. (2004) observed that knee extension during the front foot contact phase (front foot contact until ball release) was linked to higher peak braking forces. In particular, the use of an already extended, or extending front knee was linked to both increased peak ground reaction forces and increased loading rates. Significant moderate correlations were observed between the amount of knee extension during the front foot contact phase and the time to peak vertical force $(r=-0.41, P<0.01)$ as well as time to peak braking force $(r=-0.41, P<0.01)$. The angle of the front knee at the instant of ball release was also observed to be significantly correlated with peak vertical $(r=0.31, P<0.05)$ and peak braking $(r=$ $0.38, \mathrm{P}<0.05)$ ground reaction forces. Bowlers with a straighter front knee at ball release typically had higher peak ground reaction forces.

These previous studies have documented peak ground reaction forces and loading rates as well as noting correlations with individual aspects of bowling technique. However, none has accounted for the effect of interactions between technique parameters or attempted to identify the key aspects of technique that determine the ground reaction force characteristics. The aim of the current study was to identify the aspects of fast bowlers' front leg technique that best explain the peak ground reaction forces and time to peak force during the front foot contact phase of fast bowling.

\section{METHODS}

Twenty elite male fast bowlers (mean \pm standard deviation: age $20.1 \pm 2.6$ years; height $1.88 \pm 0.08 \mathrm{~m}$; body mass $81.5 \pm 7.1 \mathrm{~kg}$ ) participated in this investigation. All bowlers were members of the England and Wales Cricket Board (ECB) elite fast bowling group - being either current England bowlers or identified as likely to play for England within the next 3 - 5 years. All bowlers were deemed fit to bowl by their County or National Team physiotherapist. The testing procedures were explained to each bowler and informed consent was obtained in accordance with the Loughborough University Ethical Advisory Committee.

All bowlers conducted a thorough warm-up prior to data collection. Each bowler performed six maximum effort deliveries of good length; these were recorded using an 18 camera (M2 MCam) Vicon Motion Analysis System (OMG PIc, Oxford, UK) operating at $300 \mathrm{~Hz}$. Ground reaction forces during the front foot contact phase of the bowling action were measured using a Kistler force plate $(900 \times 600 \mathrm{~mm}, 1008$ $\mathrm{Hz}$ ). The force plate was built into the indoor testing facility and had a layer of 
artificial grass $(25 \mathrm{~mm})$ on its surface. The indoor cricket facility allowed bowlers to use a full-length run-up on a standard sized artificial cricket pitch wearing their own indoor bowling footwear.

Forty-seven $14 \mathrm{~mm}$ retro-reflective markers were attached to each subject (Figure 1), positioned over bony landmarks. An additional marker, a $15 \times 15 \mathrm{~mm}$ patch of 3M Scotch-Lite reflective tape, was attached to the ball to enable the instant of ball release and the speed of the ball to be determined. The ankle, knee, shoulder, elbow and wrist joint centres were calculated from a pair of markers placed across the joint, positioned such that their mid-point coincided with the joint centre (Ranson et al., 2009). The hip joint centres were calculated from markers placed over the left and right anterior superior iliac spine and the left and right posterior superior iliac spine (Davis et al., 1991). Lower and upper trunk motions were defined using the four markers on the pelvis in addition to markers placed over the cephalad and caudal ends of the sternum as well as the spinous processes of L1, T10 and C7 (Ranson et al., 2009). Anthropometric measurements were taken in accordance with the geometric model of Yeadon (1990), enabling subject-specific segmental mass and segmental mass centre locations to be determined throughout the bowling action for each bowler. This enabled the position of the centre of mass of the whole body to be calculated.

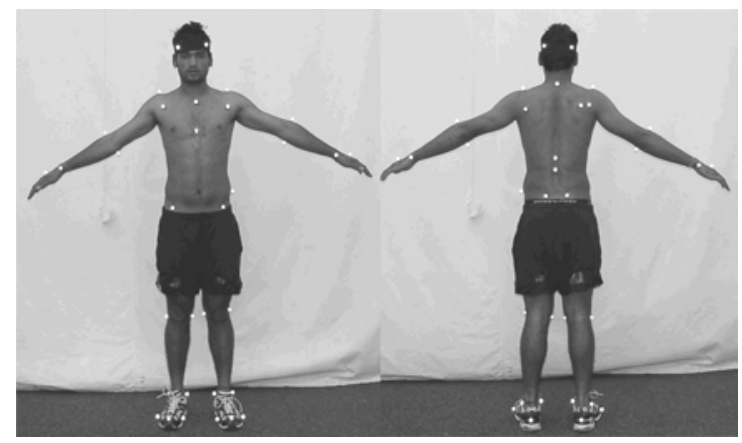

Figure 1. Marker locations.

Three bowling trials for each bowler (maximum effort deliveries with good Vicon data) were manually labelled and processed within Vicon's software (OMG Plc, Oxford, UK). The instants of back foot contact, front foot contact and front foot flat were identified using the motions of the markers on the foot. Ground contact was defined as the first frame where the motion of the markers on the foot was visually observed to change due to contact with the ground. Front foot flat corresponded to the first frame where the forefoot was on the ground. Ball release was identified using the time-history of the distance between the ball marker and the mid-point of a pair of markers placed across the wrist. The frame corresponding to ball release was defined as the first frame where this distance increased by more than $20 \mathrm{~mm}$ relative to the distance in the previous image. All marker trajectories were filtered using a recursive fourth-order low-pass Butterworth filter with a cut-off frequency of $30 \mathrm{~Hz}$ (determined using the methodology of Winter, 1990). This cut-off frequency was chosen to ensure that marker trajectories were not overly smoothed during the impact phases.

A global coordinate system was defined with the y-axis pointing down the wicket (towards the batsman), the x-axis pointing to the right and the z-axis corresponding to the upwards vertical. Three-dimensional orthogonal local reference frames were defined describing an 18 segment representation of the body consisting of a: head and neck; upper trunk; lower trunk; pelvis; 2 x upper-arm; 2 x lower-arm; 2 x hand; 2 
$x$ upper-leg; $2 \times$ lower-leg; and $2 \times$ two-segment foot. These local reference frames were defined using three markers on the segment itself, enabling segment orientations and joint angles to be calculated. The origins were located at the lower joint centre of the segment, when standing in an anatomical position. The z-axis pointed upwards along the longitudinal axis of the segment, the $x$-axis pointed to the subject's right (flexion-extension axis of the joint) and the $y$-axis pointed forwards.

Joint angles were calculated as Cardan angles, defining the rotation applied to the parent coordinate system (proximal segment) in order to bring it into coincidence with the coordinate system of the child segment (distal segment). Rotation angles were calculated using an xyz sequence - representing an initial rotation about the $x$ axis of the parent, followed by rotation about a floating $y$-axis of the parent and finally the z-axis of the child. These rotations corresponded to flexion-extension, abductionadduction, and longitudinal rotation, respectively (Cole et al., 1993).

Eight kinematic parameters were calculated for each trial, describing the run-up and front leg technique: run-up speed (horizontal); 'plant angle'; 'initial foot angle', knee angle and hip angle (at front foot contact); ankle dorsi-flexion, knee flexion and hip flexion (front foot contact until ball release). Angles describing the ankle (foot flat $\approx 90^{\circ}$ ), knee $\left(\right.$ straight $=180^{\circ}$, flexed $\left.<180^{\circ}\right)$ and hip $\left(\right.$ straight $=180^{\circ}$, flexed $\left.<180^{\circ}\right)$ corresponded to the anatomical flexion/extension angle of the joint with joint flexion represented by a decrease in the joint angle; the amount of flexion was calculated during the period from front foot contact until ball release. In order to account for differences in the orientation of the foot at front foot contact, an 'initial foot angle' was calculated for each trial, rather than using the ankle angle. This initial foot angle was the angle between the global $y$-axis (the line pointing down the wicket) and a line joining a projection of the ankle and MTP joint centres onto a vertical global plane (Figure 2a). Similarly, the 'plant angle' was calculated by projecting the hip and ankle joint centres onto a vertical plane. The plant angle being the angle between the downwards vertical and a line joining these two projected points (Figure 2b). The front leg classification, in accordance with the system defined by Portus et al. (2004) was calculated from the knee flexion / extension time history of each bowling trial. A 'trunk alignment angle', describing the orientation of the trunk relative to the front leg at front foot contact, was determined by projecting the mid-point of the shoulders and the joint centres of the hip and ankle of the front leg onto a vertical plane and calculating the angle between them.

The horizontal run-up speed (global y-direction) was calculated as the mean velocity of the mass centre over a period of 18 frames $(0.060 \mathrm{~s})$ immediately prior to the instant of back foot contact. Four kinetic parameters were also defined: peak forces in the vertical and horizontal directions (Figure 3); and the times from front foot contact until the occurrence of these peak forces. Peak forces were normalised using the bowler's body weight (BW).

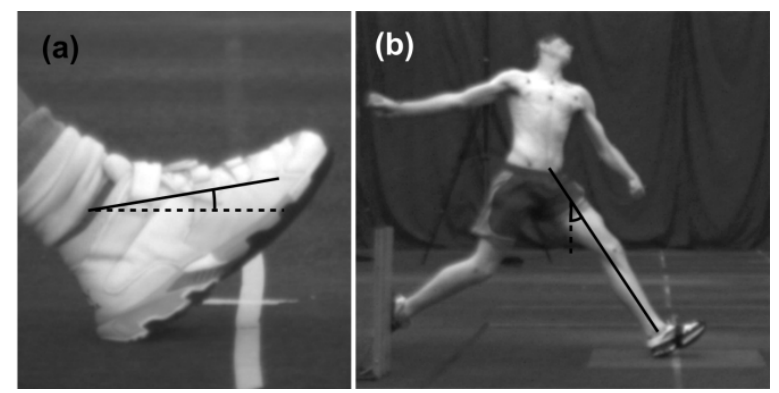

Figure 2. (a) The foot angle and (b) the plant angle at the instant of front foot contact. 


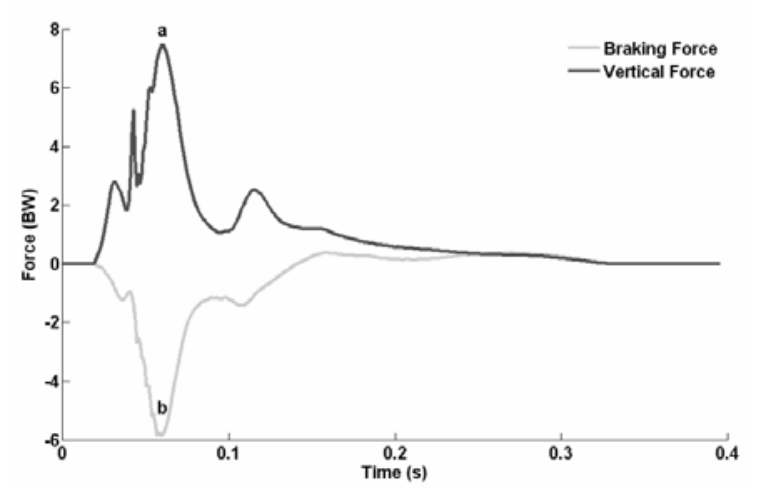

Figure 3. A typical ground reaction force trace during the front foot contact phase, with (a) the peak vertical force and (b) the peak horizontal (braking) force indicated.

All statistical analyses were performed within SPSS v.17 (SPSS Corporation, USA). The variation observed in each parameter from the three trials (eight kinematic parameters and four kinetic parameters) was assessed using an analysis of variance (ANOVA). The between-trial standard deviation of the observations ranged from $8.8-20.4 \%$ (mean 12.8\%) of the between-bowler values for the parameters calculated in this study. This corresponds to an Intra-Class Correlation Coefficient (ICC) of $0.96-0.99$ (mean 0.98) and indicates good between-trial repeatability for all parameters calculated (Norton et al., 2000). As a consequence, it was appropriate to average the three trials analysed for each parameter to provide representative data for each bowler. To address the aim of the study and identify which of the eight kinematic parameters (independent variables) best explained the four kinetic parameters (dependent variables), forwards stepwise linear regression was used with the requirement for the inclusion of a parameter in the regression equation being $\mathrm{P}<0.10$. To confirm the normality of the residuals in each regression model featured within this paper Kolmogorov-Smirnov tests for normality were carried out. The P-values ranged from 0.47 to 0.97 indicating no evidence against the assumption of normality of the residuals. To help explain the results obtained the plant angle at FFC was correlated against the trunk alignment angle at FFC. To enable comparison with previous studies, the bowlers were split into four groups according to the front leg techniques defined by Portus et al. (2004) and the mean and standard deviations for each parameter (kinetic and kinematic) were compared using the Kruskall-Wallis test.

\section{RESULTS}

The twenty bowlers participating in this study released the ball at speeds of 32.8 - 39.7 m.s-1 (mean $34.9 \pm 1.7$ m.s-1) (Table I). There was substantial variation in the peak vertical and horizontal forces (Table II) with a mean peak vertical ground reaction force of $6.7 \pm 1.4 \mathrm{BW}$ and a peak braking force of $4.5 \pm 0.8 \mathrm{BW}$. Using the front knee technique classification system introduced by Portus et al. (2004), the bowlers were classified as: nine flexor-extenders; six flexors; three extenders; and two using a constant brace. Splitting the kinetic parameters into the Portus et al. (2004) derived classifications (Table II) and comparing the mean and standard deviation across the four groups revealed that there were no significant differences between the groups. 
The best individual predictor of peak vertical force was the initial foot angle that accounted for $30 \%$ of the variation observed $(P<0.05$, Table III). This increased to $63 \%$ with the addition of plant angle and run-up speed into the regression equation. Higher peak vertical ground reaction forces were associated with a smaller initial foot angle, a smaller plant angle and a quicker run-up speed. Again splitting the kinematic predictors into the Portus et al. (2004) derived classifications and comparing the mean and standard deviation of each group for each predictor revealed that there were no significant differences between the groups.

Table I. Summary of technique parameters

\begin{tabular}{lll}
\hline technique parameter & range & mean \pm SD \\
\hline run-up speed $\left({\left.\mathrm{m} . \mathrm{s}^{-1}\right)}^{-1}\right.$ & $4.77-6.76$ & $5.79 \pm 0.58$ \\
foot angle at FFC & $-31^{\circ}-11^{\circ}$ & $-8^{\circ} \pm 13^{\circ}$ \\
knee angle at FFC & $148^{\circ}-173^{\circ}$ & $164^{\circ} \pm 6^{\circ}$ \\
hip angle at FFC & $117^{\circ}-149^{\circ}$ & $133^{\circ} \pm 9^{\circ}$ \\
plant angle at FFC & $27^{\circ}-43^{\circ}$ & $36^{\circ} \pm 4^{\circ}$ \\
ankle dorsi-flexion: FFC to BR & $4^{\circ}-26^{\circ}$ & $12^{\circ} \pm 6^{\circ}$ \\
knee flexion: FFC to BR & $0^{\circ}-45^{\circ}$ & $17^{\circ} \pm 11^{\circ}$ \\
hip flexion: FFC to BR & $10^{\circ}-44^{\circ}$ & $25^{\circ} \pm 10^{\circ}$ \\
\hline
\end{tabular}

Note. FFC $=$ front foot contact, $\mathrm{BR}=$ ball release

Table II. Summary of kinetic parameters

\begin{tabular}{lcccccc}
\hline kinetic parameters & range & mean \pm SD & $\begin{array}{c}\text { braced } \\
\text { mean } \pm \text { SD }\end{array}$ & $\begin{array}{c}\text { flexor } \\
\text { mean } \pm \text { SD }\end{array}$ & $\begin{array}{c}\text { flex. }- \text { ext. } \\
\text { mean } \pm \text { SD }\end{array}$ & $\begin{array}{c}\text { extender } \\
\text { mean } \pm \text { SD }\end{array}$ \\
\hline peak vertical force (BW) & $4.0-8.6$ & $6.7 \pm 1.4$ & $6.7 \pm 0.6$ & $6.8 \pm 1.5$ & $6.8 \pm 1.8$ & $6.3 \pm 0.6$ \\
peak braking force (BW) & $2.6-6.1$ & $4.5 \pm 0.8$ & $4.7 \pm 0.5$ & $4.2 \pm 0.9$ & $4.5 \pm 0.9$ & $4.6 \pm 0.1$ \\
time to peak vertical force (s) & $0.01-0.05$ & $0.03 \pm 0.01$ & $0.03 \pm 0.00$ & $0.03 \pm 0.01$ & $0.03 \pm 0.01$ & $0.03 \pm 0.01$ \\
time to peak braking force (s) & $0.02-0.05$ & $0.03 \pm 0.01$ & $0.03 \pm 0.00$ & $0.03 \pm 0.01$ & $0.03 \pm 0.01$ & $0.03 \pm 0.01$ \\
\hline
\end{tabular}

Table III. Regression equations for peak force using stepwise linear regression

\begin{tabular}{|c|c|c|c|c|}
\hline & parameter(s) & coefficient & $\mathrm{P}$ & $\begin{array}{l}\text { percentage } \\
\text { explained }\end{array}$ \\
\hline \multicolumn{5}{|c|}{ peak vertical force: } \\
\hline 1 & initial foot angle & -0.059 & 0.013 & 30 \\
\hline 2 & $\begin{array}{l}\text { initial foot angle } \\
\text { plant angle }\end{array}$ & $\begin{array}{l}-0.052 \\
-0.124\end{array}$ & $\begin{array}{l}0.022 \\
0.089\end{array}$ & 41 \\
\hline 3 & $\begin{array}{l}\text { initial foot angle } \\
\text { plant angle } \\
\text { run-up speed }\end{array}$ & $\begin{array}{l}-0.072 \\
-0.211 \\
1.416\end{array}$ & $\begin{array}{l}0.001 \\
0.004 \\
0.007\end{array}$ & 63 \\
\hline \multicolumn{5}{|c|}{ peak braking force: } \\
\hline 1 & run-up speed & 0.428 & 0.155 & N/A \\
\hline 2 & $\begin{array}{l}\text { run-up speed } \\
\text { initial foot angle }\end{array}$ & $\begin{array}{c}0.691 \\
-0.028\end{array}$ & $\begin{array}{l}0.027 \\
0.038\end{array}$ & 31 \\
\hline
\end{tabular}

Note: $\mathrm{P}<0.10$ indicates a significant relationship 
There was no significant correlation between any individual technique parameter and the peak braking force; run-up speed was the parameter closest to being significant $(P=0.155$, Table III). However, $31 \%$ of the variation in peak braking force was explained when run-up speed and the initial foot angle were entered into the regression equation together. There was insufficient evidence to support the addition of any further technique parameters into the regression equation (i.e. $P>0.10)$.

The variation in the time to peak vertical/horizontal force were best explained by the initial foot angle with $45 \%$ (vertical, $\mathrm{P}<0.05$ ) and $63 \%$ (horizontal, $\mathrm{P}<0.05$ ) of the variation being accounted for (Table IV). In both cases the percentage variation explained was increased (to $55 \%$ and $75 \%$, respectively) by the addition of the amount of knee flexion, between front foot contact and ball release, into the regression equation. Those bowlers with the longest time until peak force had the largest initial foot angle and flexed their front knee less.

Table IV. Regression equations for time to peak force using stepwise linear regression

\begin{tabular}{|c|c|c|c|c|}
\hline & parameter(s) & coefficient & $\mathrm{P}$ & $\begin{array}{c}\text { percentage } \\
\text { explained }\end{array}$ \\
\hline \multicolumn{5}{|c|}{ time to peak vertical force: } \\
\hline 1 & initial foot angle & 0.001 & 0.001 & 45 \\
\hline 2 & $\begin{array}{l}\text { initial foot angle } \\
\text { knee flexion }\end{array}$ & $\begin{array}{c}0.001 \\
-0.000\end{array}$ & $\begin{array}{l}0.000 \\
0.074\end{array}$ & 55 \\
\hline \multicolumn{5}{|c|}{ time to peak braking force: } \\
\hline 1 & initial foot angle & 0.001 & 0.000 & 63 \\
\hline 2 & $\begin{array}{l}\text { initial foot angle } \\
\text { knee flexion }\end{array}$ & $\begin{array}{c}0.001 \\
-0.000\end{array}$ & $\begin{array}{l}0.000 \\
0.012\end{array}$ & 75 \\
\hline
\end{tabular}

The plant angle at front foot contact was significantly correlated with trunk alignment angle at front foot contact; $r=-0.644, P<0.05$ ). In particular, bowlers whose front leg and trunk are more aligned with each other at front foot contact (larger trunk alignment angle), typically have a smaller plant angle at front foot contact (Figure 4a) while bowlers with a larger plant angle at front foot contact (Figure $4 \mathrm{~b}$ ), tend to have their trunk less aligned with the front leg (smaller trunk alignment angle) at front foot contact.

(a)

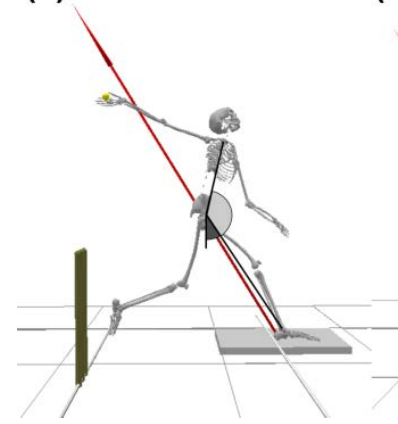

(b)

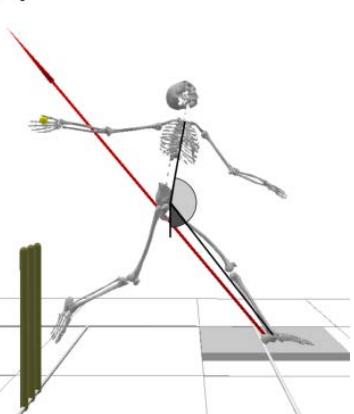

Figure 4. The resultant ground reaction force vector during the initial part of the front foot contact phase for (a) a bowler with a small plant angle and (b) a bowler with a large plant angle. In both graphics the plant angle (darker shading) and trunk alignment angle (lighter shading) are shown. 


\section{DISCUSSION}

The current study has identified the aspects of fast bowlers' front leg technique that best explain the peak ground reaction forces and time to peak force during the front foot contact phase of fast bowling. In particular, $63 \%$ of the variation in peak vertical ground reaction force observed between bowlers can be explained using just three kinematic parameters: initial foot angle; plant angle; and run-up speed. Two of these parameters, run-up speed and initial foot angle, were also the best predictors of the peak braking force (explaining 31\% of the variation observed). The front knee classification system used by Portus et al. (2004) was not significantly related to the peak ground reaction forces or the time to peak force during the front foot contact phase of fast bowling. This is most likely to be due to the Portus et al. (2004) classification system considering changes in knee angle between front foot contact and ball release, while peak ground reaction forces occur in the first $50 \mathrm{~ms}$ of the front foot contact phase of the bowling action (Table II).

The initial foot angle has not been calculated previously in the literature yet it was the best individual predictor of peak vertical force. A smaller initial foot angle (i.e. in bowlers who first contact the ground with the mid-foot rather than heel strike) was associated with higher peak forces in both the vertical and braking directions. Visual inspection of the data collected suggested the majority of bowlers experienced peak ground reaction forces a few frames after their forefoot made contact with the ground. It should therefore be expected that those bowlers with a larger initial foot angle (i.e. a heel strike technique) will experience lower peak forces (as the impact is spread over a longer period of time) and these forces will take a longer time to reach a peak value relative to front foot contact. Future studies could investigate this relationship further, in particular why peak forces only occur once the forefoot has made contact with the ground.

The link between a larger plant angle at front foot contact and lower peak vertical ground reaction forces (Table III) can be potentially explained through geometry. The resultant ground reaction force was roughly aligned with the front leg during the initial part of the front foot contact phase (Figure 4) for all bowlers. Consequently a larger plant angle (Figure $4 b$ ) is associated with a lower peak vertical force as the vertical component of the ground reaction force is smaller. The relationship between plant angle and peak vertical force may also be partly attributable to the relationship between plant angle and the 'trunk alignment angle' (Figure 4). It is speculated that having the front leg and trunk more aligned (smaller plant angle; Figure 4a) results in a greater reaction force acting up through the body due to there being less hip flexion during the initial part of front foot contact. This potential relationship should be further investigated in the future to understand the mechanics in more detail.

A quicker run-up speed was associated with higher peak forces in both the vertical and braking directions. Bowlers with a quicker run-up have more linear momentum as they strike the ground at front foot contact and would be expected to generate higher peak forces as the bowler rapidly slows the linear velocity of their centre of mass. These results support the suggestion of Hurrion et al. (2000) that the higher peak braking forces reported in more recent studies may be partly attributable to quicker run-up speeds.

The relatively low percentage variation explained for the peak braking force (31\% using two parameters) is partly attributable to the strong correlation between peak braking force and peak vertical force $(r=0.666, P=0.001)$. Some bowlers have large peak ground reaction forces and others have far smaller peak ground reaction forces. It was observed that peak vertical force alone could explain $44 \%$ of 
the variation in peak braking force. The best individual predictor of the time to peak force, in both the vertical and braking directions, was the initial foot angle. Those bowlers with a larger initial foot angle (i.e. a more pronounced heel strike technique) took a longer time to reach their peak force. As discussed previously, the majority of bowlers experienced peak ground reaction forces a few frames after their forefoot made contact with the ground. Consequently, the additional time taken for bowlers with a larger initial foot angle to reach peak force can be considered to be approximately the delay between the instant of front foot contact and the instant the forefoot hit the ground.

The amount of knee flexion was also observed to be a significant explanatory parameter for the time to peak force, with increased knee flexion being associated with a shorter time to peak force. These results appear to directly contradict those of Portus et al. (2004) who reported that bowlers who used a technique where the front knee extends, or was already extended, had a shorter time to peak force. When considering these results it is important to remember that in both studies the knee flexion or extension was calculated during the period from front foot flat until ball release, however, peak ground reaction force occurs very early within this period. In the current study, nine of the bowlers tested used a flexor-extender front knee technique (at least $10^{\circ}$ of knee flexion prior to $10^{\circ}$ or more knee extension). On closer examination of the knee angles in the current study, it was observed that the majority of bowlers extended their front knee during the first few frames of the front foot contact phase. As the ground reaction force increased most bowlers began to flex their knee. These results suggest that the bowlers may have attempted to maintain an extended front knee throughout the front foot contact phase, but many were unable to withstand the forces exerted by the ground and flexed their front knee as a result. In this light, the knee flexion observed in bowlers appears to be as a consequence of the shorter time to peak force, as opposed to the motion of the knee determining the delay as has been previously shown.

Small sample sizes are a common problem when studying elite populations; the sample of 20 elite fast bowlers used in the current study represents a relatively large sample for this particular population. Although the sample size limits the power of the statistical tests that can be conducted, there is sufficient data to enable regression to be used to identify those parameters that best explain the variation in peak ground reaction forces. This represents an important tool in this type of situation, enabling the most important technique variables to be identified. It is important with multiple regression not to attribute too much meaning to the order of entry of variables, their marginal contributions to the R2, or to their relative significance levels. In this paper multiple regression analysis has been used appropriately, alongside more traditional biomechanical research evidence, and this has led to insights that would not be achieved using simple correlations. The significance levels required for inclusion of technique parameters in the regression equation were relaxed to $10 \%(P<0.10)$ in order to increase the ability of the tests to detect relationships of potential interest.

The results provide a relationship between fast bowling technique and ground reaction forces and are likely to provide a valuable framework to improve the understanding of fast bowling. The natural progression of this study is to investigate the relationship between ground reaction forces and loading within the body (e.g. in the lower back) along with a comparison of ground reaction forces when bowling indoors in training footwear versus bowling outdoors in bowling boots. Potential links between ground reaction forces, technique and common injuries to fast bowlers such as lower back stress fractures and ankle joint posterior impingement should also be investigated. A key issue raised by this study is the relationship between ground 
reaction forces and fast bowling performance as it is currently unclear whether high ground reaction forces are required to bowl fast. As the links between ground reaction forces and both injuries and performance are not yet clear, no direct recommendations can be made with regard to the coaching of fast bowlers at this point. However, this study does clearly demonstrate the importance of technique factors on ground reaction forces.

In conclusion, peak forces and time to peak force were found to vary widely among the group of elite fast bowlers analysed. These variations in peak force and time to peak force were linked to the orientation of the front leg at the instant of front foot contact. In particular, bowling techniques incorporating a larger plant angle and a heel strike technique were associated with lower peak forces and longer times to peak force during the front foot contact phase, which may help reduce the likelihood of lower back injuries. It also appears that the flexion of the front knee during the front foot contact phase, occurring in the majority of bowlers, is a consequence of the high peak ground reaction forces. To understand the mechanics of these relationships in more detail, future research studies could use simulation modelling to investigate the effect of changing front leg technique on the ground reaction forces for individual bowlers.

\section{ACKNOWLEDGEMENTS}

This project was funded by the England and Wales Cricket Board (ECB).

\section{REFERENCES}

Bartlett, R.M., Stockill, N.P., Elliott, B.C., \& Burnett, A.F. (1996). The biomechanics of fast bowling in men's cricket: A review. Journal of Sports Sciences, 14, 403424.

Brooks, J.H., Fuller, C.W., Kemp, S.P., \& Reddin, D.B. (2005). Epidemiology of injuries in English professional rugby union: part 1 match injuries. British Journal of Sports Medicine, 39, 757-766.

Burnett, A., Barrett, C., Marshall, R., Elliott, B.C., \& Day, R. (1998). Threedimensional measurement of lumbar spine kinematics for fast bowlers in cricket. Clinical Biomechanics, 13, 574-583.

Burnett, A., O'Sullivan, P., Ankarberg, L., Gooding, M., Nelis, R., Offermann, F., \& Persson, J. (2008). Lower lumbar spine axial rotation is reduced in end-range sagittal postures when compared to a neutral spine posture. Manual Therapy, 13, 300-306.

Cole, G.K., Nigg, B.M., Ronsky, J.L. \& Yeadon, M.R. (1993). Application of the joint coordinate system to three-dimensional joint attitude and movement representation: A standardization proposal. Journal of Biomechanical Engineering, 115, 344-349.

Davis, R.B., Ounpuu, S., Tyburski, D., \& Gage, J.R. (1991). A gait analysis data collection and reduction technique. Human Movement Science, 10, 575-587.

Elliott, B.C. (2000). Back injuries and the fast bowler in cricket. Journal of Sports Sciences, 18, 983-991.

Foster, D., John, D., Elliott, B., Ackland, T., \& Fitch, K. (1989). Back injuries to fast bowlers in cricket: A prospective study. British Journal of Sports Medicine, 23, 150-154. 
Hall, M.C. (1999). Ground reaction forces experienced at front foot impact in cricket pace bowling. Unpublished undergraduate dissertation, Liverpool John Moores University, Liverpool.

Hurrion, P.D., Dyson, R., \& Hale, T. (2000). Simultaneous measurement of back and front foot ground reaction forces during the same delivery stride of the fastmedium bowler. Journal of Sports Sciences, 18, 993-997.

Mansingh, A., Harper, L., Headley, S., King-Mowatt, J., \& Mansingh, G. (2006). Injuries in West Indies cricket 2003-2004. British Journal of Sports Medicine, 40, 119-123.

Mason, B.R., Weissensteiner, J.R., \& Spence, P.R. (1989). Development of a model for fast bowling in cricket. Excel, 6, 2-12.

Norton, K., Marfell-Jones, M., Whittingham, N., Kerr, D., Carter, L., Saddington, K., \& Gore, C. (2000). Anthropometric assessment protocols. In C. Gore, (Ed), Physiological tests for elite athletes (p. 66-85). Champaign, IL: Human Kinetics Publishers.

Orchard, J, \& Seward, H. (2002). Epidemiology of injuries in the Australian Football League, seasons 1997-2000. British Journal of Sports Medicine, 36, 39-44.

Orchard, J.W., James, T., \& Portus, M.R. (2006). Injuries to elite male cricketers in Australia over a 10-year period. Journal of Science and Medicine in Sport, 9, 459467.

Portus, M.R., Mason, B.R., Elliott, B.C., Pfitzner, M.C., \& Done, R.P. (2004). Technique factors related to ball release speed and trunk injuries in high performance cricket fast bowlers. Sports Biomechanics, 3, 263-283.

Ranson, C.A., Burnett, A.F., King, M., Patel, N., \& O'Sullivan, P.B. (2008). The relationship between bowling action classification and three-dimensional lower trunk motion in fast bowlers in cricket. Journal of Sports Sciences, 26, $267-276$.

Ranson, C., King, M., Burnett, A., Worthington, P. and Shine, K. (2009). The effect of coaching intervention on elite fast bowling technique over a two year period. Sports Biomechanics, 8, 261-274.

Winter, D.A., 1990. Biomechanics and Motor Control of Human Movement. New York: Wiley.

Yeadon, M.R. (1990). The simulation of aerial movement - II: A mathematical inertia model of the human body. Journal of Biomechanics, 23, 67-74. 